\title{
The finite speed of propagation for solutions to stochastic viscoelastic wave equation
}

Fei Liang ${ }^{1^{*}}$ and Zhe Hu${ }^{1}$

\author{
"Correspondence: \\ fliangmath@126.com; \\ liangfei@xust.edu.cn \\ 'Department of Mathematics, Xi An \\ University of Science and \\ Technology, Xi An, China
}

\section{Abstract}

In this paper, a class of second order stochastic evolution equations with memory

$$
u_{t t}(t, x)-\Delta u(t, x)+\int_{0}^{t} g(t-s) \Delta u(s, x) d s+f(u)=\sigma(u) \frac{\partial W(x, t)}{\partial t}, \quad x \in D \subset \mathbb{R}^{n},
$$

is considered, where $f$ is a continuous function with polynomial growth of order less than or equal to $n /(n-2)$ and $\sigma$ is Lipschitz with $\sigma(0)=0$. By Tartar's energy method, we prove that for any solution to the equation the propagate speed is finite.

MSC: $60 \mathrm{H} 15 ; 35 \mathrm{~L} 05 ; 35 \mathrm{~L} 70$

Keywords: Stochastic evolution equations; The finite speed of propagation; Multiplicative noise

\section{Introduction}

In this paper, we consider the stochastic viscoelastic wave equations

$$
\left\{\begin{array}{l}
u_{t t}(t, x)-\Delta u(t, x)+\int_{0}^{t} g(t-s) \Delta u(s, x) d s+f(u)=\sigma(u) \frac{\partial W(x, t)}{\partial t}, \\
\quad x \in D, t \in(0, T), \\
u(x, t)=0, \quad x \in \partial D, t \in(0, T), \\
u(x, 0)=u_{0}(x), \quad u_{t}(x, 0)=v_{0}(x), \quad x \in D,
\end{array}\right.
$$

where $D$ is a bounded domain in $\mathbb{R}^{n}$ with a smooth boundary $\partial D, g$ is the relaxation function satisfying the hyperbolicity and the existence condition, i.e.

(G1) $g \geq 0 \in C^{1}[0, \infty)$ is a non-increasing function satisfying

$$
\int_{0}^{\infty} g(s) d s=1-l>0
$$

$f: \mathbb{R} \rightarrow \mathbb{R}$ satisfies

(A1) $f$ is a continuous function, $f(s) s \geq 0, \forall s \in \mathbb{R}$ and

$$
|f(s)| \leq C\left(1+|s|^{p+1}\right), \quad \forall s \in \mathbb{R},
$$


where $p$ satisfies

$$
\begin{cases}0 \leq p \leq \frac{2}{n-2}, & \text { if } n>2 \\ p \geq 0, & \text { if } n=1,2\end{cases}
$$

$\{W(t, x): t \geq 0\}$ is a $H$-valued $R$-Wiener process on the probability space with the variance operator $R$ satisfying $\operatorname{Tr} R<\infty$.

When $g(t)=0,(1.1)$ becomes a nonlinear wave equation. Marinelli and Quer-Sarsanyons [1] proved existence of weak solutions in the probabilistic sense for a general class of stochastic semilinear wave equations on bounded domains of $\mathbb{R}^{n}$ driven by a possibly discontinuous square integrable martingale. Under a more restrictive condition on $f$, Barbu and Röckner [2] found that the propagation speed of the solutions of (1.1) with dissipative damping is finite by Tartar's energy method. The result is similar to the classical finite speed of propagation result for the solution to the Klein-Gordon equation.

When $g(t) \neq 0$, for the current equation (1.1), the memory part makes it difficult to estimate the energy by using these methods. Hence, Wei and Jiang [3] studied (1.1) with $\sigma \equiv 1$ in another way. They showed the existence and uniqueness of solution for (1.1) and obtained the decay estimate of the energy function of the solution. In [4], Liang and Guo obtained asymptotic stability and extend the decay estimate of [3] for the general equation (1.1) with multiplicative noise. Moreover, Liang and Gao [5] also obtained the existence and uniqueness of global mild solutions for (1.1) driven by Lévy noise.

In this paper, we prove that, for any solution to (1.1) with probability one, the speed of propagation is with velocity less than or equal to 1 . This localization result is new for the case of the second order stochastic evolution equations with memory we consider here. The standard strategy to prove this property for the deterministic Klein-Gordon equation is based on the Paley-Wiener theorem combined with point arguments [6]. However, for the current equation (1.1), the memory part makes it difficult to use these methods. So we shall use a different approach, inspired by Tartar's energy method [7].

This paper is organized as follows. In Sect. 2 we present some assumptions needed for our work and give the existence theorem for a unique global weak solution. Section 3 is devoted to the proof of the finite propagate speed.

\section{Preliminaries}

Set $H=L^{2}(D)$ and $V=H_{0}^{1}(D)$ with norm denoted by $\|\cdot\|$ and $\|\nabla \cdot\|$, respectively. In addition, both $H$ and $V$ are Hilbert spaces if we endow them with the usual inner products $(\cdot, \cdot)$ and $\langle\cdot, \cdot\rangle$, respectively. Let $\mathscr{H}=V \times H$ with the norm $\|U\|_{\mathscr{H}}=\left(\|\nabla u\|^{2}+\|v\|^{2}\right)^{\frac{1}{2}}$ for any $U=(u, v) \in \mathscr{H}$.

Let $(\Omega, P, \mathcal{F})$ be a complete probability space for which a $\left\{\mathcal{F}_{t}, t \geq 0\right\}$ filtration of sub- $\sigma$ fields of $\mathcal{F}$ is given. A point of $\Omega$ will be denoted by $\omega$ and $\mathbf{E}(\cdot)$ stands for the expectation with respect to probability measure $P$. Suppose that $\{W(t, x): t \geq 0\}$ is a $H$-valued $Q$ Wiener process on the probability space with the covariance operator $Q$ satisfying $\operatorname{Tr} Q<$ $\infty$. It has mean $\mathbf{E} W(x, t)=0$ and satisfies

$$
\mathbf{E}[(W(t), \varphi) \wedge(W(s), \psi)]=(t \wedge s)(Q \varphi, \psi)
$$


for any $\varphi, \psi \in H$. Moreover, we can assume that $Q$ has the following form:

$$
Q e_{i}=\lambda_{i} e_{i}, \quad i=1,2, \ldots
$$

where $\lambda_{i}$ are eigenvalues of $Q$ satisfying $\sum_{i=1}^{\infty} \lambda_{i}<\infty$ and $\left\{e_{i}\right\}$ are the corresponding eigenfunctions with $c_{0}:=\sup _{i \geq 1}\left\|e_{i}\right\|_{\infty}<\infty$ (where $\|\cdot\|_{\infty}$ denotes the super-norm). In this case,

$$
W(t, x)=\sum_{i=1}^{\infty} \sqrt{\lambda_{i}} B_{i}(t) e_{i}
$$

where $\left\{B_{i}(t)\right\}$ is a sequence of independent copies of standard Brownian motions in one dimension.

Now, we give the definition of solution to $(1.1)$; see $[8,9]$ for details. For the definition of a solution, we assume that

$$
\left(u_{0}, u_{1}\right) \in H_{0}^{1}(D) \times L^{2}(D) .
$$

Definition 2.1 ([8,9]) Under the assumption $(2.1), u$ is said to be a solution of $(1.1)$ on the interval $[0, T]$ if

$$
\begin{aligned}
& \left(u, u_{t}\right) \text { is } H_{0}^{1}(D) \times L^{2}(D) \text {-valued progressively measurable, } \\
& \left(u, u_{t}\right) \in L^{2}\left(\Omega ; C\left([0, T] ; H_{0}^{1}(D) \times L^{2}(D)\right)\right), \quad \text { for almost all } \omega, \\
& u(0)=u_{0}, \quad u_{t}(0)=u_{1}, \\
& u_{t t}-\Delta u+\int_{0}^{t} g(t-\tau) \Delta u(\tau) d \tau+f(u)=\sigma(u) \partial_{t} W(t, x)
\end{aligned}
$$

hold in the sense of distributions over $(0, T) \times D$ for almost all $\omega$.

Remark 2.1 Equations (2.3) and (2.5) imply that

$$
\begin{aligned}
\left(u_{t}(t), \phi\right)= & \left(u_{1}, \phi\right)-\int_{0}^{t}(\nabla u, \nabla \phi) d s+\int_{0}^{t}(f(u), \phi) d s \\
& +\int_{0}^{t}\left(\int_{0}^{s} g(s-\tau) \nabla u(\tau) d \tau, \nabla \phi(s)\right) d s+\int_{0}^{t}\left(\phi, \sigma(u) d W_{s}\right),
\end{aligned}
$$

for all $t \in[0, T]$ and all $\phi \in H_{0}^{1}(D)$. In fact, (2.6) is a conventional form for the definition of solution to stochastic differential equations. Here we say $u$ is a strong solution of Eq. (1.1).

As regards the existence equation (1.1), moreover, we assume $f$ also satisfies:

(A2) Assume that, for every $N>0$, there exists constant $L_{f}(N)$ such that, for all $t \geq 0$ and all $u, v \in V$ with $\|\nabla u\| \leq N$ and $\|\nabla v\| \leq N$,

$$
\|f(u)-f(v)\|^{2} \leq L_{f}(N)\|\nabla(u-v)\|^{2} .
$$

Remark 2.2 ([8,9]) It is clear that $f(u)=|u|^{p} u$ satisfies conditions (A1) and (A2), where $p$ satisfies (1.3). 
Under our hypotheses on $g$ and $f$, we can derive an unique maximal local mild solution to (1.1) by following the arguments of [9] almost step by step.

Theorem 2.1 Assume that (G1), (A1) and (A2) are satisfied and $\left(u_{0}(x), u_{1}(x)\right): \Omega \rightarrow \mathscr{H}$ be $\mathcal{F}_{0}$-measurable. Then (1.1) admits a unique local mild solution $u \in C^{1}\left(\left[0, \tau_{\infty}\right) \times D ; H\right) \cap$ $C\left(\left[0, \tau_{\infty}\right) ; V\right)$ satisfying

$$
\lim _{t \rightarrow \tau_{\infty}} \sup \|\nabla u\|=+\infty
$$

and

$$
u\left(t \wedge \tau_{k}\right)=S\left(t \wedge \tau_{k}\right) u_{0}+\int_{0}^{t \wedge \tau_{k}} S(\tau) u_{1} d \tau-\int_{0}^{t \wedge \tau_{k}} 1 * S\left(t \wedge \tau_{k}-\tau\right) f(u) d \tau+I_{\tau_{k}}(\sigma)\left(t \wedge \tau_{k}\right)
$$

for all $t>0$ and $k \in \mathbb{N}$, where $\tau_{\infty}$ is a stopping time defined by

$$
\begin{aligned}
& \tau_{\infty}=\lim _{k \rightarrow \infty} \tau_{k} \quad \text { with } \tau_{k}=\inf \{t \geq 0 ;\|\nabla u\| \geq k\}, \\
& I_{\tau_{k}}(\sigma)(t)=\int_{0}^{t} 1_{\left[0, \tau_{k}\right)}(\tau) 1 * S(t-\tau) \sigma\left(u\left(\tau \wedge \tau_{k}\right), u_{t}\left(\tau \wedge \tau_{k}\right)\right) d W(\tau),
\end{aligned}
$$

and $S(t)$ is the resolvent operator for the equation

$$
u_{t t}-\Delta u+\int_{0}^{t} g(t-\tau) \Delta u(\tau) d \tau=0
$$

Moreover, if $u_{0} \in H^{2}(D) \cap V$ and $u_{1} \in V$, then the mild solution of (1.1) is a strong solution and belongs to $C^{1}\left(\left[0, \tau_{\infty}\right) \times D ; H^{2}(D) \cap V\right)$.

Define the energy functional $\mathcal{E}(t)$ associated with our system (1.1)

$$
\mathcal{E}(u(t))=\left\|u_{t}(t)\right\|^{2}+\left(1-\int_{0}^{t} g(s) d s\right)\|\nabla u(t)\|^{2}+(g \circ \nabla u)(t),
$$

where

$$
(g \circ w)(t)=\int_{0}^{t} g(t-s)\|w(t)-w(s)\|^{2} d s .
$$

Theorem 2.2 Assume that (G1), (A1) and (A2) are satisfied and $u(0)=\left(u_{0}(x), u_{1}(x)\right)$ : $\Omega \rightarrow \mathscr{H}$ be $\mathcal{F}_{0}$-measurable. Let $u$ be the unique local mild solution to problem (1.1) with life span $\tau_{\infty}$, then $\tau_{\infty}=\infty \mathbb{P}$-a.s.

Proof First, we consider the case of $\mathcal{E}(u(0))<\infty$. Let $u(t), 0 \leq t<\tau_{\infty}$, be a maximal local mild solution to problem (1.1). Define a sequence of stopping times by

$$
\tau_{k}=\inf \{t \geq 0:\|\nabla u\| \geq k\}, \quad k \geq 1 .
$$

By Theorem 2.1, then $\lim _{k \rightarrow \infty} \tau_{k}=\tau_{\infty}$. For any $t \geq 0$, we will show that $u\left(t \wedge \tau_{k}\right) \rightarrow u(t)$ a.s. as $k \rightarrow \infty$, so that the local solution becomes a global one. To this end, it suffices to show that $\tau_{k} \rightarrow \infty$ as $k \rightarrow \infty$ with probability one. 
Now one of the main obstacles is that the solution $u$ to problem (1.1) may have only a finite lifespan, i.e., $\tau_{\infty}<\infty$. For this purpose, we fix $k \in \mathbb{N}$ and introduce the following function:

$$
\tilde{f}(t)=1_{\left[0, \tau_{k}\right)}(t) f\left(u\left(t \wedge \tau_{k}\right)\right), \quad \tilde{\sigma}(t)=1_{\left[0, \tau_{k}\right)}(t) \sigma\left(u\left(t \wedge \tau_{k}\right)\right), \quad t \geq 0 .
$$

One can see that the processes $\tilde{f}$ and $\tilde{\sigma}$ are bounded. We consider the following linear nonhomogeneous stochastic equation:

$$
\left\{\begin{array}{l}
v_{t t}=\Delta v-\int_{0}^{t} g(t-s) \Delta v(s) d s-\tilde{f}(t)+\tilde{\sigma}(t) \frac{\partial W(x, t)}{\partial t}, \quad t>0 \\
v(x, 0)=u_{0}(x), \quad v_{t}(x, 0)=u_{1}(x)
\end{array}\right.
$$

By Theorem 3.1 of [3], there exists a unique global mild solution of (2.8) with the form

$$
\begin{aligned}
v(t)= & S(t) u_{0}+\int_{0}^{t} S(\tau) u_{1} d \tau-\int_{0}^{t} 1 * S(t-\tau) \tilde{f}(\tau) d \tau \\
& +\int_{0}^{t} 1 * S(t-\tau) \tilde{\sigma}(\tau) d W(\tau), \quad t \geq 0 .
\end{aligned}
$$

Hence the stopped process $v\left(\cdot \wedge \tau_{k}\right)$ satisfies

$$
\begin{aligned}
v\left(t \wedge \tau_{k}\right)= & S\left(t \wedge \tau_{k}\right) u_{0}+\int_{0}^{t \wedge \tau_{k}} S(s) u_{1} d s-\int_{0}^{t \wedge \tau_{k}} 1 * S\left(t \wedge \tau_{k}-s\right) \tilde{f}(s) d s \\
& +I_{\tau_{k}}(\tilde{\sigma})\left(t \wedge \tau_{k}\right), \quad t \geq 0,
\end{aligned}
$$

where

$$
I_{\tau_{k}}(\tilde{\sigma})(t)=\int_{0}^{t} 1_{\left[0, \tau_{k}\right)}(s) 1 * S(t-s) \tilde{\sigma}(s) d W(s, x) .
$$

One can observe that (see [10])

$$
\begin{aligned}
I_{\tau_{k}}(\tilde{\sigma})(t) & =\int_{0}^{t} 1_{\left[0, \tau_{k}\right)}(s) 1 * S(t-s) \tilde{\sigma}(s) d W(s, x) \\
& =\int_{0}^{t} 1_{\left[0, \tau_{k}\right)}(s) 1 * S(t-s) \sigma\left(u\left(t \wedge \tau_{k}\right)\right) d W(s, x)=I_{\tau_{k}}(\sigma)(t), \quad t \geq 0 .
\end{aligned}
$$

Therefore, for every $k \geq 1$, from Theorem 2.1, we have

$$
\begin{aligned}
v\left(t \wedge \tau_{k}\right)= & S\left(t \wedge \tau_{k}\right) u_{0}+\int_{0}^{t \wedge \tau_{k}} S(s) u_{1} d s-\int_{0}^{t \wedge \tau_{k}} 1 * S\left(t \wedge \tau_{k}-s\right) \tilde{f}(s) d s+I_{\tau_{k}}(\tilde{\sigma})\left(t \wedge \tau_{k}\right) \\
= & S\left(t \wedge \tau_{k}\right) u_{0}+\int_{0}^{t \wedge \tau_{k}} S(s) u_{1} d s-\int_{0}^{t \wedge \tau_{k}} 1 * S\left(t \wedge \tau_{k}-s\right) \tilde{f}(s) d s+I_{\tau_{k}}(\sigma)\left(t \wedge \tau_{k}\right) \\
= & S\left(t \wedge \tau_{n}\right) u_{0}+\int_{0}^{t \wedge \tau_{n}} S(s) u_{1} d s+I_{\tau_{k}}(\sigma)\left(t \wedge \tau_{k}\right) \\
& -\int_{0}^{t \wedge \tau_{k}} 1 * S\left(t \wedge \tau_{k}-s\right) 1_{\left[0, \tau_{k}\right)}(s) f\left(u\left(s \wedge \tau_{k}\right)\right) d s
\end{aligned}
$$




$$
\begin{aligned}
= & S\left(t \wedge \tau_{k}\right) u_{0}+\int_{0}^{t \wedge \tau_{k}} S(\tau) u_{1} d \tau \\
& -\int_{0}^{t \wedge \tau_{k}} 1 * S\left(t \wedge \tau_{k}-\tau\right) f(u) d \tau+I_{\tau_{k}}(\sigma)\left(t \wedge \tau_{k}\right) \\
= & u\left(t \wedge \tau_{k}\right), \quad \mathbb{P} \text {-a.s. } t \geq 0 .
\end{aligned}
$$

By virtue of the Itô rule for $\left\|v_{t}\left(t \wedge \tau_{k}\right)\right\|^{2}$, we have

$$
\begin{aligned}
\left\|v_{t}\left(t \wedge \tau_{k}\right)\right\|^{2}= & \left\|u_{1}\right\|^{2}+\left\|\nabla u_{0}\right\|^{2}-\left\|\nabla v\left(t \wedge \tau_{k}\right)\right\|^{2} \\
& +2 \int_{0}^{t \wedge \tau_{k}}\left\langle\int_{0}^{s} g(s-r) v(r) d r, v_{t}(s)\right\rangle d s-2 \int_{0}^{t \wedge \tau_{k}}\left(v_{t}, \tilde{f}(s)\right) d s \\
& +\int_{0}^{t \wedge \tau_{k}}\left(v_{t}(s), \tilde{\sigma}(s) d W(x, s)\right)+\int_{0}^{t \wedge \tau_{k}} \operatorname{Tr}\left(Q^{1 / 2} \tilde{\sigma}^{*}(s) \tilde{\sigma}(s) Q^{1 / 2}\right) d s .
\end{aligned}
$$

(Note that we could only use the Itô formula on a strong solution, we can approximate the energy function of a mild solution $v$ by a sequence of energy functions such that the corresponding strong solution sequence $\left\{v_{m}\right\}$ converges to $v$; see Theorem 3.1 for details.) Using the condition (G1), we have

$$
\begin{aligned}
& \left\langle\int_{0}^{s} g(s-r) v(r) d r, v_{t}(s)\right\rangle \\
& \quad=\int_{0}^{s} g(s-r) \int_{D} \nabla v_{t}(s) \cdot \nabla v(r) d r d x \\
& \quad=\int_{0}^{s} g(s-r) \int_{D} \nabla v_{t}(s)(\nabla v(r)-\nabla v(s)) d x d r+\int_{0}^{s} g(s-r) \int_{D} \nabla v_{t}(s) \nabla v(s) d x d r \\
& \quad=-\frac{1}{2} \int_{0}^{s} g(s-r) \frac{d}{d s} \int_{D}|\nabla v(r)-\nabla v(s)|^{2} d x d r+\frac{1}{2} \int_{0}^{s} g(r) \frac{d}{d s} \int_{D}|\nabla v(s)|^{2} d x d r \\
& \quad=\frac{1}{2} \frac{d}{d s}\left(\int_{0}^{s} g(r) d r\|\nabla v(s)\|^{2}-(g \circ \nabla v)(s)\right)+\frac{1}{2}\left(g^{\prime} \circ \nabla v\right)(s)-\frac{1}{2} g(s)\|\nabla v(s)\|^{2} \\
& \leq \frac{1}{2} \frac{d}{d s}\left(\int_{0}^{s} g(r) d r\|\nabla v(s)\|^{2}-(g \circ \nabla v)(s)\right),
\end{aligned}
$$

which implies that

$$
\begin{aligned}
& 2 \int_{0}^{t \wedge \tau_{k}}\left\langle\int_{0}^{s} g(s-r) v(r) d r, v_{t}(s)\right\rangle d s \\
& \quad \leq \int_{0}^{t \wedge \tau_{k}} g(r) d r\left\|\nabla v\left(t \wedge \tau_{k}\right)\right\|^{2}-(g \circ \nabla v)\left(t \wedge \tau_{k}\right) .
\end{aligned}
$$

By the definition of $\tilde{f}$ and $\tilde{\sigma}$, we have

$$
\begin{aligned}
2 \int_{0}^{t \wedge \tau_{k}}\left(v_{t}, \tilde{f}(s)\right) d s & =2 \int_{0}^{t \wedge \tau_{k}}\left(v_{t}(s), 1_{\left[0, \tau_{k}\right)}(s) f\left(u\left(s \wedge \tau_{k}\right)\right)\right) d s \\
& =2 \int_{0}^{t \wedge \tau_{k}}\left(v_{t}(s), f(u(s))\right) d s
\end{aligned}
$$


and

$$
\begin{aligned}
& \int_{0}^{t \wedge \tau_{k}} \operatorname{Tr}\left(Q^{1 / 2} \tilde{\sigma}^{*}(s) \tilde{\sigma}(s) Q^{1 / 2}\right) d s \\
& \quad=\int_{0}^{t \wedge \tau_{k}} \sum_{i \in I}\left(\tilde{\sigma}^{*}(s) \tilde{\sigma}(s) Q^{1 / 2} e_{i}, Q^{1 / 2} e_{i}\right) \\
& \quad=\int_{0}^{t \wedge \tau_{k}} \sum_{i \in I}\left\|\tilde{\sigma}^{*}(s) Q^{1 / 2} e_{i}\right\|^{2} d s \leq c_{0}^{2} \operatorname{Tr} Q \int_{0}^{t \wedge \tau_{k}}\|\sigma(u(s))\|^{2} d s .
\end{aligned}
$$

Substituting (2.11), (2.12) and (2.13) into (2.9) and taking the expectation to both sides, we obtain

$$
\begin{aligned}
\mathbb{E} \mathcal{E}\left(v\left(t \wedge \tau_{k}\right)\right) \leq & \mathbb{E} \mathcal{E}(v(0))-2 \mathbb{E} \int_{0}^{t \wedge \tau_{k}}\left(v_{t}(s), f(u(s))\right) d s \\
& +c_{0}^{2} \operatorname{Tr} Q \mathbb{E} \int_{0}^{t \wedge \tau_{k}}\|\sigma(u(s))\|^{2} d s,
\end{aligned}
$$

where we also use the definition of $\mathcal{E}(t)$. Recalling $v(t)=u(t)$ for $t \leq \tau_{k}$, from (2.14) we get

$$
\begin{aligned}
& \mathbb{E} \mathcal{E}\left(u\left(t \wedge \tau_{k}\right)\right) \\
& \leq \mathbb{E} \mathcal{E}(u(0))-2 \mathbb{E} \int_{0}^{t \wedge \tau_{k}}\left(u_{t}(s), f(u(s))\right) d s+c_{0}^{2} \operatorname{Tr} Q \mathbb{E} \int_{0}^{t \wedge \tau_{k}}\|\sigma(u(s))\|^{2} d s \\
& \leq \mathbb{E} \mathcal{E}(u(0))-2 \int_{0}^{t}\left(u_{t}\left(s \wedge \tau_{k}\right), f\left(u\left(s \wedge \tau_{k}\right)\right)\right) d s+c_{0}^{2} \operatorname{Tr} Q \mathbb{E} \int_{0}^{t}\left\|\sigma\left(u\left(s \wedge \tau_{k}\right)\right)\right\|^{2} d s \\
& \leq \mathbb{E} \mathcal{E}(u(0))-2 \mathbb{E} \int_{D} F\left(u\left(t \wedge \tau_{k}\right)\right) d x+2 \mathbb{E} \int_{D} F\left(u_{0}\right) d x \\
& \quad+C \int_{0}^{t} \mathbb{E}\left(\left(\left\|\nabla u\left(s \wedge \tau_{n}\right)\right\|^{2}+\left\|u_{t}\left(s \wedge \tau_{n}\right)\right\|^{2}\right)\right) d s \\
& \leq \mathbb{E} \mathcal{E}(u(0))-2 \mathbb{E} \int_{D} F\left(u\left(t \wedge \tau_{k}\right)\right) d x+2 \mathbb{E} \int_{D} F\left(u_{0}\right) d x \\
& \left.\quad+L_{\sigma} \int_{0}^{t} \mathbb{E} \mathcal{E}\left(u\left(s \wedge \tau_{n}\right)\right)\right) d s,
\end{aligned}
$$

where $F(s)=\int_{0}^{s} f(\tau) d \tau$ and $C$ is a positive constant. From (A1), we have $F(s) \geq 0$ for $s \in \mathbb{R}$. So using the Gronwall inequality to (2.15), we have

$$
\mathbb{E} \mathcal{E}\left(u\left(t \wedge \tau_{k}\right)\right) \leq\left(C_{1}+\mathbb{E} \mathcal{E}(u(0))\right) e^{C t}
$$

for any $k \geq 1$ and $t \geq 0$, where $C_{1}=2 \mathbb{E} \int_{D} F\left(u_{0}\right) d x$. It follows that

$$
\begin{aligned}
\mathbb{P}\left(\left\{\tau_{k}<t\right\}\right) & =\mathbb{E} 1_{\left\{\tau_{k}<t\right\}}=\int_{\Omega} \frac{\left\|\nabla u\left(\tau_{k}\right)\right\|^{2}}{\left\|\nabla u\left(\tau_{k}\right)\right\|^{2}} 1_{\left\{\tau_{k}<t\right\}} d \mathbb{P} \\
& \leq \frac{1}{l\left\|\nabla u\left(\tau_{k}\right)\right\|^{2}} \int_{\Omega} \mathcal{E}\left(u\left(t \wedge \tau_{k}\right)\right) 1_{\left\{\tau_{k}<t\right\}} d \mathbb{P} \\
& \leq \frac{1}{l k^{2}} \mathbb{E} \mathcal{E}\left(u\left(t \wedge \tau_{k}\right)\right) \leq \frac{1}{l k^{2}}\left(C_{1}+\mathbb{E} \mathcal{E}(u(0))\right) e^{C t}
\end{aligned}
$$


where $l$ is defined in (G1). Since $\mathcal{E}(u(0))<\infty$, the above inequality gives $\mathbb{P}\left(\left\{\tau_{k}<t\right\}\right) \leq \frac{C_{t}}{k^{2}}$, which, with the aid of the Borel-Cantelli lemma implies that $\mathbb{P}\left(\left\{\tau_{\infty}<t\right\}\right)=0$ or $\tau_{\infty}=\infty$, $\mathbb{P}$-a.s.

Therefore, Theorem 2.2 holds under the additional condition $\mathcal{E}(u(0))<\infty$. In fact, we can get a unique global mild solution to (1.1) for the deterministic initial condition $u(0)=\left(u_{0}(x), u_{1}(x)\right) \in \mathcal{H}$. Consequently, for any Borel probability measure $\mu$ on $\mathcal{H}$ there exists a martingale solution to (1.1) with the initial condition $\mu$ by [11]. Using pathwise uniqueness and a suitable version of the Yamada-Watanabe theory (see [12], Theorem 2) we find a unique global mild solution to (1.1) for every $\mathcal{F}_{0}$-measurable initial condition $u(0): \Omega \rightarrow \mathcal{H}$.

\section{The finite speed of propagation}

In this section, we will apply Tartar's energy method to show that for any solution to Eq. (1.1) the propagate speed is finite. Let $K$ is a closed subset of $D$ and denote by $d_{K}(x)$ the distance from $x \in D$ to $K$, i.e.

$$
d_{K}(x)=\inf \left\{|x-y|_{d} ; y \in K\right\} .
$$

For any $r>0$, we set

$$
K_{r}=\left\{x \in K ; d_{K}(x) \leq r\right\}
$$

For a given function $\varphi: D \rightarrow \mathbb{R}$, let the support $\{\varphi\}$ denote the closure of the set $\{x \in$ $D ; \varphi(x) \neq 0\}$. Then we have the following.

Theorem 3.1 Assume that (G1) and (A1) hold. Let $1 \leq d<\infty$ and $K$ be a closed subset of D. Let $u(t)$ be any solution to (1.1) with initial data $u_{0}(x) \in V$ and $v_{0}(x) \in H$. If

$$
\operatorname{support}\left\{u_{0}(x)\right\} \subset K, \quad \operatorname{support}\left\{v_{0}(x)\right\} \subset K,
$$

then $\mathbb{P}$-a.s.

$$
\operatorname{support}\{u(x, t)\} \subset K_{t}, \quad \forall t \geq 0 .
$$

Proof Define a $C^{1}$ function $\rho$ such that

$$
\begin{aligned}
& \rho(0)=0, \quad \forall s \leq 0, \quad \rho(s)>0, \quad \forall s>0, \\
& \rho^{\prime}(s) \geq 0, \quad \forall s \leq 0, \\
& \sup _{s \geq 0}\left(\rho(s)+\rho^{\prime}(s)\right)<\infty .
\end{aligned}
$$

We consider the local energy function $\phi:[0, \infty) \times V \times H \rightarrow \mathbb{R}$ defined by

$$
\begin{aligned}
\phi(t, u, v)= & \frac{1}{2} \int_{D} \rho\left(d_{K}(x)-t\right) \\
& \times\left[\left(1-\int_{0}^{t} g(s) d s\right)|\nabla u|^{2}+|v|^{2}+\int_{0}^{t} g(t-s)|\nabla u(s)-\nabla u(t)|^{2} d s\right] d x .
\end{aligned}
$$


From (3.1) and (3.3), we have

$$
\phi(0, u, v)=\phi\left(0, u_{0}, v_{0}\right)=0 .
$$

Note that we could only use the Itô formula on a strong solution to Eq. (1.1), we can approximate the energy function of a mild solution $u$ by a sequence of energy functions such that the corresponding strong solution sequence $\left\{u_{m}\right\}$ converges to $u$. Set

$$
A=-\Delta, \quad R(m ; A)=(m I-A)^{-1},
$$

then $D(A)=H^{2}(D) \cap H_{0}^{1}(D)$ and $R(m ; A)$ is bounded by $1 / m$. Let

$$
u_{m}(t)=R(m ; A) u(t), \quad v_{m}=R(m ; A) v(t), \quad m \geq 1 .
$$

From (1.1), $\left(u_{m}(t), v_{m}(t)\right)$ satisfies

$$
\left\{\begin{aligned}
d u_{m}(t)= & v_{m}(t) d t \\
d v_{m}(t)= & -A u_{m} d t+\int_{0}^{t} g(t-\tau) A u_{m}(\tau) d \tau d t-R(m ; A) f(u(t)) d t \\
& +R(m ; A) \sigma(u) \frac{\partial W(x, t)}{\partial t}, \\
u_{m 0}(x)= & R(m ; A) u_{0}(x), \quad v_{m 0}(x)=R(m ; A) v_{0}(x) .
\end{aligned}\right.
$$

Since $u(t)$ is a solution to (1.1), from Definition 2.1, we have

$$
u_{m}(t) \in C\left([0, T] ; L^{2}(\Omega, D(A))\right), \quad v_{m}(t) \in C\left([0, T] ; L^{2}(\Omega, V)\right) .
$$

In addition, by the Sobolev embedding theorem and condition (A1), we have $f(u) \in$ $L^{2}\left([0, T] ; L^{2}(\Omega \times D)\right)$, which implies

$$
R(m ; A) f(u) \in L^{2}\left([0, T] ; L^{2}(\Omega, D(A))\right), \quad \forall m \geq 1 .
$$

Applying the Itô formula to $\phi\left(t, u_{m}, v_{m}\right)$, we get

$$
\begin{aligned}
d \phi\left(t, u_{m}, v_{m}\right)= & \phi_{t}^{\prime}\left(t, u_{m}, v_{m}\right) d t+\left\langle\phi_{u_{m}}^{\prime}\left(t, u_{m}, v_{m}\right), v_{m}\right\rangle d t+\left(\phi_{v_{m}}^{\prime}\left(t, u_{m}, v_{m}\right), v_{m}^{\prime}(t)\right) d t \\
& +\frac{1}{2} \sum_{i=1}^{\infty} \lambda_{i}\left(\phi_{v_{m} v_{m}}^{\prime \prime}\left(t, u_{m}, v_{m}\right) R(m ; A) \sigma(u) e_{i}, R(m ; A) \sigma(u) e_{i}\right) d t, \quad t \geq 0
\end{aligned}
$$

which implies from (3.6) and (3.7)

$$
\begin{aligned}
\phi\left(t, u_{m}, v_{m}\right)= & -\frac{1}{2} \int_{0}^{t} \int_{D} \rho^{\prime}\left(d_{K}(x)-s\right)\left[\left(1-\int_{0}^{s} g(\tau) d \tau\right)\left|\nabla u_{m}(s)\right|^{2}+\left|v_{m}(s)\right|^{2}\right. \\
& \left.+\int_{0}^{s} g(s-\tau)\left|\nabla u_{m}(\tau)-\nabla u_{m}(s)\right|^{2} d \tau\right] d x d s \\
& -\frac{1}{2} \int_{0}^{t} \int_{D} g(s) \rho\left(d_{K}(x)-s\right)\left|\nabla u_{m}(s)\right|^{2} d x d s \\
& +\frac{1}{2} \int_{0}^{t} \int_{D} \rho\left(d_{K}(x)-s\right) \int_{0}^{s} g^{\prime}(s-\tau)\left|\nabla u_{m}(\tau)-\nabla u_{m}(s)\right|^{2} d \tau d x d s
\end{aligned}
$$




$$
\begin{aligned}
& +\int_{0}^{t} \int_{D} \rho\left(d_{K}(x)-s\right)\left(1-\int_{0}^{s} g(\tau) d \tau\right) \nabla u_{m}(s) \cdot \nabla v_{m}(s) d x d s \\
& -\int_{0}^{t} \int_{D} \rho\left(d_{K}(x)-s\right) \int_{0}^{s} g(s-\tau)\left(\nabla u_{m}(\tau)-\nabla u_{m}(s)\right) \cdot \nabla v_{m}(s) d \tau d x d s \\
& -\int_{0}^{t} \int_{D} \rho\left(d_{K}(x)-s\right) R(m ; A) f(u(s)) v_{m}(s) d x d s \\
& +I_{1}+I_{2}+\int_{0}^{t}\left(\rho\left(d_{K}(x)-s\right) v_{m}, R(m ; A) \sigma(u) d W(s)\right) \\
& +\frac{1}{2} \sum_{i=1}^{\infty} \lambda_{i} \int_{0}^{t} \int_{D} \rho\left(d_{K}(x)-s\right)\left|R(m ; A) \sigma(u) e_{i}\right|^{2} d x d s
\end{aligned}
$$

where

$$
\begin{aligned}
& I_{1}=\int_{0}^{t} \int_{D} \rho\left(d_{K}(x)-s\right) \Delta u_{m}(s) v_{m}(s) d x d s \\
& I_{2}=-\int_{0}^{t} \int_{D} \rho\left(d_{K}(x)-s\right) v_{m}(s) \int_{0}^{s} g(s-\tau) \Delta u_{m}(\tau) d \tau d x d s .
\end{aligned}
$$

Taking into account that

$$
\left|d_{K}(x)-d_{K}(y)\right| \leq|x-y|_{d} \quad \text { for all } x, y \in \mathbb{R}^{d},
$$

we infer that $d_{K} \in W^{1, \infty}\left(\mathbb{R}^{d}\right)$ and

$$
\left|\nabla d_{K}(x)\right| \leq 1, \quad \text { a.e. } x \in \mathbb{R}^{d} \text {. }
$$

By Green's formula we have

$$
\begin{aligned}
I_{1}= & -\int_{0}^{t} \int_{D} \rho\left(d_{K}(x)-s\right) \nabla u_{m}(s) \cdot \nabla v_{m}(s) d x d s \\
- & \int_{0}^{t} \int_{D} \rho^{\prime}\left(d_{K}(x)-s\right) v_{m}(s) \nabla u_{m}(s) \cdot \nabla d_{K}(s) d x d s, \\
I_{2}= & \int_{0}^{t} \int_{D} \rho\left(d_{K}(x)-s\right) \int_{0}^{s} g(s-\tau) \nabla u_{m}(\tau) \cdot \nabla v_{m}(s) d \tau d x d s \\
& +\int_{0}^{t} \int_{D} \rho^{\prime}\left(d_{K}(x)-s\right) v_{m}(s) \int_{0}^{s} g(s-\tau) \nabla u_{m}(\tau) \nabla d_{K}(x) d \tau d x d s \\
= & \int_{0}^{t} \int_{D} \rho\left(d_{K}(x)-s\right) \nabla u_{m}(s) \cdot \nabla v_{m}(s) d \int_{0}^{s} g(\tau) d \tau d x d s \\
& +\int_{0}^{t} \int_{D} \rho\left(d_{K}(x)-s\right) \int_{0}^{s} g(s-\tau)\left(\nabla u_{m}(\tau)-\nabla u_{m}(s)\right) \cdot \nabla v_{m}(s) d \tau d x d s \\
& +\int_{0}^{t} \int_{D} \rho^{\prime}\left(d_{K}(x)-s\right) v_{m}(s) \int_{0}^{s} g(s-\tau) \nabla u_{m}(\tau) \nabla d_{K}(x) d \tau d x d s .
\end{aligned}
$$


Substituting (3.11) and (3.12) into (3.9), we get

$$
\begin{aligned}
\phi\left(t, u_{m}, v_{m}\right)= & -\frac{1}{2} \int_{0}^{t} \int_{D} \rho^{\prime}\left(d_{K}(x)-s\right)\left[\left(1-\int_{0}^{s} g(\tau) d \tau\right)\left|\nabla u_{m}(s)\right|^{2}+\left|v_{m}(s)\right|^{2}\right. \\
& +2 v_{m}(s) \nabla u_{m}(s) \cdot \nabla d_{K}(s)+\int_{0}^{s} g(s-\tau)\left|\nabla u_{m}(\tau)-\nabla u_{m}(s)\right|^{2} d \tau \\
& \left.-2 v_{m}(s) \int_{0}^{s} g(s-\tau) \nabla u_{m}(\tau) \nabla d_{K}(x) d \tau\right] d x d s \\
& -\frac{1}{2} \int_{0}^{t} \int_{D} g(s) \rho\left(d_{K}(x)-s\right)\left|\nabla u_{m}(s)\right|^{2} d x d s \\
& +\frac{1}{2} \int_{0}^{t} \int_{D} \rho\left(d_{K}(x)-s\right) \int_{0}^{s} g^{\prime}(s-\tau)\left|\nabla u_{m}(\tau)-\nabla u_{m}(s)\right|^{2} d \tau d x d s \\
& -\int_{0}^{t} \int_{D} \rho\left(d_{K}(x)-s\right) \frac{\partial}{\partial s} R(m ; A) F(u(s)) d x d s \\
& +\int_{0}^{t}\left(\rho\left(d_{K}(x)-s\right) v, R(m ; A) \sigma(u(s)) d W(s)\right) \\
& +\frac{1}{2} \sum_{i=1}^{\infty} \lambda_{i} \int_{0}^{t} \int_{D} \rho\left(d_{K}(x)-s\right)\left|R(m ; A) \sigma(u) e_{i}\right|^{2} d x d s .
\end{aligned}
$$

In virtue of (G1) and (3.13), it follows that

$$
\begin{aligned}
\phi\left(t, u_{m}, v_{m}\right)= & -\frac{1}{2} \int_{0}^{t} \int_{D} \rho^{\prime}\left(d_{K}(x)-s\right)\left[\left(1-\int_{0}^{s} g(\tau) d \tau\right)\left|\nabla u_{m}(s)\right|^{2}+\left|v_{m}(s)\right|^{2}\right. \\
& +2 v_{m}(s) \nabla u_{m}(s) \cdot \nabla d_{K}(s)+\int_{0}^{s} g(s-\tau)\left|\nabla u_{m}(\tau)-\nabla u_{m}(s)\right|^{2} d \tau \\
& \left.-2 v_{m}(s) \int_{0}^{s} g(s-\tau) \nabla u_{m}(\tau) \nabla d_{K}(x) d \tau\right] d x d s \\
& -\int_{0}^{t} \int_{D} \rho\left(d_{K}(x)-s\right) \frac{\partial}{\partial s} R(m ; A) F(u(s)) d x d s \\
& +\int_{0}^{t}\left(\rho\left(d_{K}(x)-s\right) v, R(m ; A) \sigma(u(s)) d W(s)\right) \\
& +\frac{1}{2} \sum_{i=1}^{\infty} \lambda_{i} \int_{0}^{t} \int_{D} \rho\left(d_{K}(x)-s\right)\left|R(m ; A) \sigma(u) e_{i}\right|^{2} d x d s .
\end{aligned}
$$

On the other hand, from (3.10) and (G1), we have

$$
\left(1-\int_{0}^{s} g(\tau) d \tau\right)\left[\left|\nabla u_{m}(s)\right|^{2}+\left|v_{m}(s)\right|^{2}+2 v_{m}(s) \nabla u_{m}(s) \cdot \nabla d_{K}(s)\right] \geq 0
$$

and

$$
\int_{0}^{s} g(s-\tau)\left[\left|\nabla u_{m}(\tau)-\nabla u_{m}(s)\right|^{2}+\left|v_{m}(s)\right|^{2}-2 v_{m}(s)\left(\nabla u_{m}(\tau)-\nabla u_{m}(s)\right) \cdot \nabla d_{K}(s)\right] d \tau \geq 0,
$$


for a.e. $x \in D, s \geq 0$. Combining (3.14)-(3.16) and taking the expectation to (3.14), we have

$$
\begin{aligned}
\mathbb{E} \phi\left(t, u_{m}, v_{m}\right) \leq & \frac{1}{2} \mathbb{E} \sum_{i=1}^{\infty} \lambda_{i} \int_{0}^{t} \int_{D} \rho\left(d_{K}(x)-s\right)\left|R(m ; A) \sigma(u) e_{i}\right|^{2} d x d s \\
& -\mathbb{E} \int_{0}^{t} \int_{D} \rho\left(d_{K}(x)-s\right) \frac{\partial}{\partial s} R(m ; A) F(u(s)) d x d s .
\end{aligned}
$$

Note that

$$
\begin{aligned}
& u_{m}(t) \rightarrow u(t) \quad \text { in } C\left([0, T] ; L^{2}(\Omega ; V)\right), \\
& v_{m}(t) \rightarrow v \quad \text { in } C\left([0, T] ; L^{2}(\Omega ; H)\right), \\
& R(m ; A) f(u) \rightarrow f(u) \quad \text { in } L^{2}\left([0, T] ; L^{2}(\Omega ; H)\right) .
\end{aligned}
$$

Then, letting $m \rightarrow \infty$ in (3.17), we obtain

$$
\begin{aligned}
\mathbb{E} \phi(t, u, v) \leq & \frac{1}{2} \mathbb{E} \sum_{i=1}^{\infty} \lambda_{i} \int_{0}^{t} \int_{D} \rho\left(d_{K}(x)-s\right)\left|\sigma(u) e_{i}\right|^{2} d x d s \\
& -\mathbb{E} \int_{0}^{t} \int_{D} \rho\left(d_{K}(x)-s\right) \frac{\partial}{\partial s} F(u(s)) d x d s .
\end{aligned}
$$

By condition (A1), we have $F(s) \geq 0$ on $\mathbb{R}$. Moreover, using (3.1), we have also $\rho\left(d_{K}(x)\right) \times$ $F\left(u_{0}\right) \equiv 0, \forall x \in D$. Then integrating (3.18) by parts, we have

$$
\mathbb{E} \phi(t, u, v) \leq \frac{1}{2} \mathbb{E} \sum_{i=1}^{\infty} \lambda_{i} \int_{0}^{t} \int_{D} \rho\left(d_{K}(x)-s\right)\left|\sigma(u) e_{i}\right|^{2} d x d s
$$

Recalling $\operatorname{Tr} Q<\infty$ and $c_{0}:=\sup _{i \geq 1}\left\|e_{i}\right\|_{\infty}<\infty$, it follows from (3.19) that

$$
\mathbb{E} \phi(t, u, v) \leq C \int_{0}^{t} \mathbb{E} \phi(s, u(s), v(s)) d s, \quad \forall t \geq 0
$$

Since $t \mapsto \phi(t, u, v)$ is continuous $\mathbb{P}$-a.s, the Gronwall inequality implies that

$$
\phi(t, u, v)=0, \quad t \geq 0, \mathbb{P} \text {-a.s. }
$$

Therefore, for any $t \geq 0, \mathbb{P}$-a.s.

$$
\rho\left(d_{K}(x)-t\right)\left[\left(1-\int_{0}^{t} g(s) d s\right)|\nabla u|^{2}+|v|^{2}+\int_{0}^{t} g(t-s)|\nabla u(s)-\nabla u(t)|^{2} d s\right]=0
$$

for $d x$-a.e. $x \in D$. Noting that (3.3) and $u(t) \in V$, we obtain $u(t, x)=0$ on $\left\{t<d_{K}(x)\right\}$ for $d x$-a.e. $x \in D$, which implies (3.2).

Remark 3.1 Note that Theorem 3.1 does not assert the existence of a solution to (1.1) with properties (3.1). It simply refers to the finite speed propagation property of solutions to (1.1). In other word, (3.2) implies that the wave front of the solution at time $t$ is in the 
neighborhood $K_{t}$ of the set $K \mathbb{P}$-a.s. This amounts to saying that any solution $u(t)$ of (1.1) propagates with finite velocity less than or equal 1 with probability 1 . The solution $u(t)$ to (1.1) has its support in the space-time cone $\left\{(t, x) \in(0, \infty) \times D ; d_{K}(x) \leq t\right\}$.

Remark 3.2 From (3.8), we see that Theorem 3.1 remains true for the stochastic viscoelastic wave equation (1.1) with nonlinear dissipative damping, i.e.

$$
u_{t t}-\Delta u+\int_{0}^{t} g(t-s) \Delta u(s)+f(u)+h\left(u_{t}\right)=\sigma(u) \frac{\partial W(x, t)}{\partial t},
$$

where $h$ is a monotonically nondecreasing $C^{1}$ function satisfying a polynomial growth condition. Leaving aside the existence problem for (3.21), we note that in this case there arises one more term

$$
-\int_{0}^{t} \int_{D} \rho\left(d_{K}(x)-s\right) v_{m} h\left(v_{m}\right) d s d x
$$

in the energy equation (3.13), which is nonpositive and so we conclude the proof as in the previous case.

\section{Acknowledgements}

The authors are indebted to the editor for giving some important suggestions which improved the presentation of this paper.

Funding

Supported in part by China NSF Grant No. 11501442, the Natural Science Basic Research Plan in Shaanxi Province of China No. 2019JM-283, and the Excellent Youth Fund of Xi An University of Science and Technology Grant Nos. 201YQ2-14, 201YQ3-12.

Availability of data and materials

No data.

Competing interests

The authors declare that they have no competing interests.

\section{Authors' contributions}

The article is a joint work of two authors who contributed equally to the final version of the paper. All authors read and approved the final manuscript.

\section{Publisher's Note}

Springer Nature remains neutral with regard to jurisdictional claims in published maps and institutional affiliations.

Received: 21 May 2019 Accepted: 12 June 2019 Published online: 08 July 2019

\section{References}

1. Marinelli, C., Quer-Sardanyons, L.: Existence of weak solutions for a class of semilinear stochastic wave equations. SIAM J. Math. Anal. 44, 906-925 (2012)

2. Barbu, V., Röckner, M.: The finite speed of propagation for solutions to nonlinear stochastic wave equations driven by multiplicative noise. J. Differ. Equ. 255, 560-571 (2013)

3. Wei, T.T., Jiang, Y.M.: Stochastic wave equations with memory. Chin. Ann. Math. 31B, 329-342 (2010)

4. Liang, F., Guo, Z.H.: Asymptotic behavior for second order stochastic evolution equations with memory. J. Math. Anal. Appl. 419, 1333-1350 (2014)

5. Liang, F., Guo, H.J.: Stochastic nonlinear wave equation with memory driven by compensated Poisson random measures. J. Math. Phys. 55, 033503 (2014)

6. Reed, M., Simon, B.: Methods of Modern Mathematical Physics II. Academic Press, New York (1975)

7. Tartar, L.: Topics in nonlinear analysis Publications Mathématiques d'Orsay, Report, Orsay (1978)

8. Liang, F., Gao, H.J.: Global existence and explosive solution for stochastic viscoelastic wave equation with nonlinear damping. Rev. Math. Phys. 26, Article ID 1450013 (2014)

9. Liang, F., Gao, H.J.: Explosive solutions of stochastic viscoelastic wave equations with damping. Rev. Math. Phys. 23, 883-902 (2011) 
10. Brzeźniak, Z., Maslowski, B., Seidler, J.: Stochastic nonlinear beam equations. Probab. Theory Relat. Fields 132, 119-149 (2005)

11. Ondreját, M.: Uniqueness for stochastic evolution equations in Banach space. Diss. Math. 426, 1-63 (2004)

12. Ondreját, M.: Brownian representation of cylindrical local martingales, martingale problem and strong Markov property of weak solutions of SPDEs in Banach space. Czechoslov. Math. J. 55, 1003-1039 (2005)

Submit your manuscript to a SpringerOpen ${ }^{\odot}$ journal and benefit from:

- Convenient online submission

Rigorous peer review

Open access: articles freely available online

- High visibility within the field

- Retaining the copyright to your article

Submit your next manuscript at $\gg$ springeropen.com 